\title{
INTERVENCIÓN PSICOLÓGICA FAMILIAR EN LA ENFERMEDAD DE ALZHEIMER
}

\author{
Balea-Fernández, Francisco Javier \\ Doctor en Psicología (USAL) \\ Doctorando en Investigación en Biomedicina (ULPGC) \\ fbalea@cop.es \\ Alonso-Ramírez, Javier \\ Doctorando en Investigación en Biomedicina (ULPGC) \\ xaalons086@gmail.com
}

Recepción Artículo: 13 marzo 2020

Admisión Evaluación: 30 marzo 2020

Informe Evaluador 1: 15 abril2020

Informe Evaluador 2: 19 abril 2020

Aprobación Publicación: 20 abril 2020

\section{RESUMEN}

La demencia es un síndrome adquirido, de naturaleza orgánica caracterizado por un deterioro permanente de la memoria y de otras funciones intelectuales, y frecuentemente acompañado de otros trastornos psiquiátricos, que ocurren sin alteración del nivel de conciencia, afectando al funcionamiento social. Las pruebas diagnósticas disponibles en la actualidad se basan fundamentalmente en criterios clínicos, apoyadas por pruebas neuropsicológicas, analíticas y de imagen. En la actualidad no se conoce el complejo proceso neurodegenerativo de esta enfermedad, pero si tenemos datos sobre la intervención de los síntomas de esta. El diseño experimental utilizado en este trabajo es de tipo "cuasiexperimental" con evaluación antes y después del programa de estimulación (taller de memoria y automatismos; lectura y lenguaje; ejercicio físico y psicomotricidad; musicoterapia y reconocimiento corporal). La muestra está formada por 21 sujetos de ambos sexos, diagnosticados de Demencia Tipo Alzheimer (DTA) probable, tratados en los centros de día y residencia durante 6 meses. La media de edad de los sujetos es de 80,71 años $\left(S_{x}=4,85\right)$. Los resultados muestran una disminución en puntuación del MEC (máximo 35 puntos) de -1,67 ( $\left.S_{x} 3.88 ; p<0.05\right)$; cuando el/la cuidadora principal acude a apoyo psicológico, la puntuación del MEC aumenta de media en $0.8\left(S_{x} 2.9\right)$, si no acude la puntuación disminuye en $3.91\left(S_{x} 3.3\right)$ con un $p$ valor de 0.03. Las conclusiones extraídas son que el tratamiento de estimulación es igual de eficaz para el mantenimiento del rendimiento cognitivo general y capacidad funcional en pacientes con deterioro cognitivo leve, moderado y grave; Cuando el trabajo se extiende al binomio familia-enfermo, mediante la aplicación de un tratamiento paralelo no farmacológico a los familiares los resultados son significativamente mejores.

Palabras clave: alzheimer; demencia; intervención psicológica familiar; estimulación; programa

\section{ABSTRACT}

Alzheimer, dementia, family psychological intervention, stimulation, program. Dementia is an acquired syndrome, of an organic nature characterized by a permanent deterioration of memory and other 


\section{INTERVENCIÓN PSICOLÓGICA FAMILIAR EN LA ENFERMEDAD DE ALZHEIMER}

intellectual functions, and frequently accompanied by other psychiatric disorders, which occur without altering the level of consciousness, affecting social functioning. Currently available diagnostic tests are primarily based on clinical criteria, supported by neuropsychological, analytical, and imaging tests. Currently, the complex neurodegenerative process of this disease is not known, but we do have data on the intervention of its symptoms. The experimental design used in this work is of a "quasi-experimental" type with evaluation before and after the stimulation program (memory and automatism workshop; reading and language; physical exercise and psychomotricity; music therapy and body recognition). The sample is made up of 21 subjects of both sexes, diagnosed with probable Alzheimer Disease, treated in the day and residence centers for 6 months. The mean age of the subjects is 80.71 years $(S D=4.85)$. The results show a decrease in MEC score (maximum 35 points) of -1.67 (SD 3.88; $p<0.05$ ); when the main caregiver goes to psychological support, the MEC score increases on average by 0.8 (SD 2.9), if he does not go, the score decreases by -3.91 (SD 3.3) with a p value of 0.03 . The conclusions drawn are that the stimulation treatment is just as effective in maintaining general cognitive performance and functional capacity in patients with mild, moderate and severe cognitive impairment; When the work extends to the family-patient binomial, by applying a parallel non-pharmacological treatment to the relatives, the results are significantly better.

Keywords: alzheimer; dementia; family psychological intervention; stimulation; program

\section{ANTECEDENTES}

Los datos sociodemográficos alertan que nunca en la historia de la humanidad se había producido un envejecimiento poblacional como el que se observa en los países desarrollados en la actualidad. Hay una estructura que va siendo cada vez más típica en los países del entorno más propiamente occidental, sobre todo el entorno europeo. Este tipo de población es la llamada "población envejecida". Ésta se define como aquella población en la que la proporción de juventud es baja, mientras que la incidencia de la población anciana es cada vez más acusada. Además, el envejecimiento de la población no sólo es consecuencia de la disminución de la fecundidad, sino que guarda una íntima relación con los índices de mortalidad cada vez más bajos, ya que la prolongación de la esperanza de vida en los países desarrollados es muy elevada (González-Rodríguez y Martín-Martín, 2002; Orduna y Naval, 2001).

Según datos de Eurostat, la esperanza de vida en España es la más alta de la Unión Europea, situándose en 83.4 años (media Europea 80.9 años) (European Observatory on Health Systems and Policies, 2019).

Desde una perspectiva demográfica, los datos extraídos del INE (Instituto Nacional de Estadística) relativos al 2017, el total de nacimientos en España fue de 391.930, mientras que el número de fallecidos fue de 423.643. Este dato indica que se producen más fallecimientos que nacimientos, por lo que la población envejece. A este dato, se une el dato de cómo está configurada la pirámide poblacional que indica que los mayores de 65 años suponen 8,7 millones y representan el 19\% de la población total (46.593.000 habitantes) (INE, 2018).

Desde una perspectiva social, el término "senilidad" y "demencia" eran términos paralelos y coincidentes, aún vigente en muchos sectores sociales. Thornton (1989) definía ambos términos como "una condición mental de la gente mayor en la cual las facultades mentales parecían haber regresado a la infancia". Además, este irreversible proceso era algo natural que ocurre con el paso de los años. De estos conceptos derivan lo que, aun en la actualidad, se conoce como "demencia senil", o incluso, la expresión peyorativa "estar senil"(Thornton, 1989).

Uno de los mayores temores en el proceso de envejecimiento es el deterioro cognitivo. Se define como "un declinar, brusco o continuo, en una o varias de las funciones intelectuales del individuo, previamente desarrolladas". La importancia de esta definición se basa en el aspecto de "desarrolladas", es decir, adquiridas. De la misma forma, deterioro cognitivo no equivale al término "demencia". En este sentido, para el diagnóstico de demencia es necesario que se cumplan una serie de criterios, entre los que se incluye el deterioro cognitivo, pero la presencia de éste no siempre implica el diagnóstico (Cubero, 2002).

Los datos sobre prevalencia e incidencia, según la Confederación Española de Alzheimer y otras demencias indican que "e/ 55\% de los mayores de 65 años que tiene demencia no está diagnosticado" (Tola, 2018). En un 
estudio realizado por Vega Alonso et al. en 2016 se determina que la prevalencia ajustada en su investigación de deterioro cognitivo leve (DCL) fue del 18,5\% (IC 95\% 17,3-19,7) de las personas mayores de 65 años, aumentando hasta el 45,3\% cuando la población estudiada es mayor de 85 años. Teniendo en cuenta los datos aportados por el anterior estudio y teniendo en cuenta que la tasa anual de progresión de DCL a demencia está entre el 8-15\% apuntando a un problema de salud comunitaria (Tola, 2018; Vega Alonso et al., 2016). En la misma línea y con similares resultados se muestra el estudio DERIVA con una prevalencia de DCL del 19\% (RodríguezSánchez et al., 2011).

En los países occidentales la Demencia Tipo Alzheimer (DTA), es la forma más frecuente de todas las demencias (mientras que, en países como Rusia o Japón, la prevalencia se inclina hacia la demencia vascular) constituyéndose entre el 50-70\% del total entre todos los subtipos de demencia (unos cuatrocientos mil enfermos en España), seguida de los cuerpos de Lewy ente el 10-30\%, demencia vascular entre 10-26\%, demencia del lóbulo frontal en $8 \%$ y la asociada a la enfermedad de Parkinson en 0.5\%. La DTA alcanza cifras en torno a los $2 / 3$ del total de las demencias (del 1\% a los 60 años, hasta un 32-38\% a los 90 años), Otra clasificación posible se basa según la posible o potencial reversibilidad o irreversibilidad: Otra de las posibles clasificaciones hace referencia a la distinción de las demencias en reversible e irreversibles. Este grupo de demencias engloba a todas aquellas potencialmente tratables 0 curables y las irreversibles o que el tratamiento se basa en paliar los síntomas y "frenar" en la medida de lo posible la enfermedad. La tercera clasificación se centra en la sintomatología, puede realizarse la diferenciación en sintomatología cortical, subcortical y mixta (Álvarez, 2002; Carrasco et al., 2019; González-Mas, 2001).

El proceso de diagnóstico de DTA comienza cuando el paciente, solo o bien con algún familiar, acude a la consulta de atención primaria con quejas de memoria y/o alteraciones en el comportamiento. En esta primera consulta, lo más frecuente es que la demencia se encuentre en fase leve/moderada (Martínez-Lage et al., 2018).

Además de los síntomas cognitivos (alteración en la memoria, gnosias, praxias, ...), en la demencia suelen padecer síntomas conductuales o no cognitivos. Estos suelen aparecer en etapas leves moderadas de la demencia, provocando gran angustia para las familias y cuidadores. Se suelen considerar como secundarios en su presentación respecto a los cognitivos, aunque los familiares más cercanos los consideran, probablemente, como los más importantes por la implicación directa en la calidad de vida del paciente, de sus familias y cuidadores. Su aparición no está ligada a la edad de presentación de la demencia, lo que indica que se relacionan con los procesos patológicos de la enfermedad en sí. Según los datos, aparecen entre un 70-90\% de los pacientes en algún momento de evolución de la demencia. Para estos síntomas no cognitivos se dispone de terapias que reducen 0 anulan estas manifestaciones del síndrome y, a veces, desaparecen espontáneamente: Trastornos afectivos (el más frecuente la depresión); Delirios (creencias falsas que se fundamentan en conclusiones incorrectas sobre la realidad); Alucinaciones (impresiones sensoriales, perceptivas, que acontecen sin que exista un estímulo real que las provoca); Apatía (desinterés por lo que le rodea); Falsos reconocimientos e identificaciones (son síntomas que están en la interfase de diferentes capacidades cognitivas, como son las agnosias y los procesos delirantes); Agitación psicomotriz (incapacidad para mantenerse quieto en un lugar determinado durante un tiempo prolongado); Alteración del patrón sueño-vigilia (consecuencia de la desorientación que sufre el paciente y la limitación 0 anulación de las actividades que realiza el paciente. La suma de los síntomas cognitivos y no cognitivos produce interacción con la relación con el entorno familiar/social. Producto de esta interacción se produce una progresiva incapacitación del paciente para realizar tanto actividades instrumentales de la vida diaria (AIVD) como actividades básicas de la vida diaria (ABVD). Las AIVD son actividades complejas como llevar la casa, las finanzas, controlar la medicación o llamar por teléfono. Las ABVD son actividades que incluyen el cuidado personal, peinarse, lavarse, comer o permanecer continente en orina y heces. Son las familias las que se hacen cargo de los cuidados de los sujetos en la mayoría de los casos (Agüera-Ortiz, 1998; Agüera et al., 2000; Carrasco et al., 2019). 


\section{INTERVENCIÓN PSICOLÓGICA FAMILIAR EN LA ENFERMEDAD DE ALZHEIMER}

\section{OBJETIVOS DE LA INVESTIGACIÓN}

El objetivo principal es verificar cuantitativamente la eficacia de la aplicación de un programa de estimulación y atención psicológica de seis meses de duración en familias que uno de los miembros tenga el diagnostico de enfermedad de Alzheimer.

\section{MUESTRA}

La muestra está formada por 21 sujetos de ambos sexos, diagnosticados de enfermedad de Alzheimer, tratados en centros de día y residencia.

En cuanto a las variables sociodemográficas: la media de edad es de 80,71 años $\left(S_{X}=4,85\right)$; sexo: varón 28.6\%; mujer 71.4\%; estado civil: soltero/a: $9.5 \%$; casado/a: $42.9 \%$; viudo/a: $47.6 \%$.

Con relación al entorno de sujeto: cuidador principal: cónyuge: 28.6\%; otro familiar: 28.6\%; residencia: 42.9\%. Familiares que acuden al programa "hablemos del Alzheimer": familiar acude: 19\%, no acude: 81\%; familiar solicita apoyo psicológico: Sí: 47.6\%; №: 52.4\%.

\section{METODOLOGÍA E INSTRUMENTOS UTILIZADOS}

El diseño utilizado en este trabajo es de tipo "cuasiexperimental". Dentro de este modelo de investigación, se encuadra en un diseño de grupo "pre y post tesf".

El programa de estimulación utilizado está basado en el manual de actividades para la intervención cognitiva de la DTA. en cuanto al planteamiento y ejecución de los diferentes talleres de estimulación cognitiva y el Programa de Psicoestimulación Integral o PPI (Boada et al., 1999; Peña-Casanova, 1999). El programa se compone de: taller de memoria y automatismos; lectura y lenguaje; ejercicio físico y psicomotricidad; musicoterapia y reconocimiento corporal. Para las familias se aplica el programa "hablemos de Alzheimer" como taller grupal con familias en las que sus familiares están en distintas fases de la enfermedad y dirigidas por un psicólogo. Además, se realiza atención psicológica personalizada a familiares que solicitan este servicio: el paradigma psicológico utilizado para la terapia individual es la Terapia Familiar Breve (De Shazer, 1989).

Para la valoración cognitiva se utiliza el Miniexamen Cognoscitivo o MEC (Lobo y Ezquerra, 1979). Se considera como variable cuantitativa discreta. Esta variable será valorada antes y después de la aplicación del programa de estimulación. Por otro lado, se contabilizan las puntuaciones parciales del MEC: puntuación total en el MEC (máximo 35); puntuación en orientación temporal (máximo 5); puntuación en orientación espacial (máximo 5); puntuación en fijación (máximo 3); puntuación en cálculo y concentración (máximo 8); puntuación en memoria (máximo 3) y puntuación en lenguaje y construcción (máximo 11).

\section{RESULTADOS}

En la tabla 1 se muestran los resultados obtenidos tras la realización de la prueba "t para muestras relacionadas". Las variables implicadas en esta prueba son las puntuaciones de las pruebas ANTES y DESPUÉS de la aplicación del programa de estimulación.

Tabla 1: resultados programa antes/después. Prueba t para muestras relacionadas.

\begin{tabular}{|l|c|c|c|c|}
\hline Diferencias entre antes-después & Media & $\begin{array}{c}\text { Desviación } \\
\text { típica }\end{array}$ & $\boldsymbol{T}$ & $\begin{array}{c}\text { Significación } \\
\text { bilateral }\end{array}$ \\
\hline MEC & $-1,67$ & 3,88 & 1,97 & 0,063 \\
\hline Orientación temporal & 0,14 & 1,39 & $-0,47$ & 0,642 \\
\hline Orientación espacial & $-0,52$ & 1,03 & 2,32 & $0,030^{*}$ \\
\hline Fijación & $-0,43$ & 0,75 & 2,36 & $0,016^{*}$ \\
\hline Cálculo y concentración & $-0,24$ & 1,70 & 0,64 & 0,528 \\
\hline Memoria & $-0,09$ & 0,77 & 0,57 & 0,576 \\
\hline Lenguaje y construcción & $-0,48$ & 1,91 & 1,14 & 0,268 \\
\hline
\end{tabular}

$* \mathrm{p}<0,05$. 
Se encuentra que existen diferencias estadísticamente significativas en los apartados de orientación espacial $(\mathrm{p}<0.05)$; fijación $(\mathrm{p}<0.05)$ y $G D S(\mathrm{p}<0.05)$ antes y después de la aplicación del programa de estimulación.

Por otro lado, se estudia si existen diferencias en el área de fijación y MEC $(p<0.05)$ dependiendo del nivel de severidad de afectación cognitiva, se observa que existen diferencias estadísticamente significativas entre el grado de severidad grave y moderado $(\mathrm{p}<0,05)$ con relación al área de fijación $(\mathrm{MEC})$, mientras que no existe diferencias estadísticamente significativas entre el grado de severidad leve y grave.

Tabla 2: DHS de Tukey en la variable severidad de afectación.

\begin{tabular}{|l|l|c|c|c|}
\hline $\begin{array}{c}\text { (I) Grado de } \\
\text { severidad }\end{array}$ & $\begin{array}{c}\text { (J) Comparación } \\
\text { grado de severidad }\end{array}$ & $\begin{array}{c}\text { Diferencia entre medias } \\
(\mathbf{I}-\boldsymbol{J})\end{array}$ & $\begin{array}{c}\text { Error } \\
\text { típ. }\end{array}$ & Significación \\
\hline \multirow{2}{*}{ Grave } & Moderada & $-0,69^{*}$ & 0,27 & 0,050 \\
\cline { 2 - 5 } & Leve & $-0,75$ & 0,31 & 0,062 \\
\hline \multirow{2}{*}{ Moderada } & Grave & $0,69^{*}$ & 0,27 & 0,050 \\
\cline { 2 - 5 } & Leve & $-0,06$ & 0,31 & 0,977 \\
\hline \multirow{2}{*}{ Leve } & Grave & 0,75 & 0,31 & 0,062 \\
\cline { 2 - 6 } & Moderada & 0,06 & 0,31 & 0,977 \\
\hline
\end{tabular}

$* \mathrm{p}<0,05$.

En la tabla 3 se muestran las puntuaciones antes y después relacionadas con las variables estudiadas.

Tabla 3: estadístico ANOVA: Test MEC y variables independientes.

\begin{tabular}{|c|c|c|c|c|c|c|c|}
\hline & & \begin{tabular}{|c|} 
Puntuación \\
Antes
\end{tabular} & \begin{tabular}{|c|} 
Puntuación \\
Después \\
\end{tabular} & Media & $\begin{array}{l}\text { Desv. } \\
\text { típica }\end{array}$ & $F$ & Sign \\
\hline \multirow{3}{*}{ MEC-Sexo } & Varón & 15,17 & 12,50 & $-2,67$ & 3,83 & \multirow{3}{*}{0,55} & \multirow{3}{*}{0,47} \\
\hline & Mujer & 17,27 & 16,00 & $-1,27$ & 3,95 & & \\
\hline & Total & 16,67 & 15,00 & $-1,67$ & 3,88 & & \\
\hline \multirow{4}{*}{$\begin{array}{l}\text { MEC- } \\
\text { Cuidador } \\
\text { principal }\end{array}$} & Cónyuge & 19,50 & 20,50 & 1,00 & 2,60 & \multirow{4}{*}{9,42} & \multirow{4}{*}{$0,02 *$} \\
\hline & \begin{tabular}{|l} 
Otro \\
familiar
\end{tabular} & 16,00 & 16,33 & 0,33 & 2,94 & & \\
\hline & Residencial & 15,22 & 10,44 & $-4,78$ & 2,95 & & \\
\hline & Total & 16,67 & 15,00 & $-1,67$ & 3,88 & & \\
\hline \multirow{3}{*}{$\begin{array}{l}\text { MEC- } \\
\text { Programa } \\
\text { "Hablemos de } \\
\text { Alzheimer". }\end{array}$} & Sí & 20,00 & 21,00 & 1,00 & 2,16 & \multirow{3}{*}{2,51} & \multirow{3}{*}{0,13} \\
\hline & No & 15,88 & 13,59 & $-2,29$ & 3,96 & & \\
\hline & Total & 16,67 & 15,00 & $-1,67$ & 3,88 & & \\
\hline \multirow{3}{*}{$\begin{array}{l}\text { MEC- “apoyo } \\
\text { psicológico" }\end{array}$} & Sí & 20,10 & 20,90 & 0,80 & 2,90 & \multirow{3}{*}{$\begin{array}{c}11,9 \\
6\end{array}$} & \multirow{3}{*}{$0,03 *$} \\
\hline & No & 13,54 & 9,63 & $-3,91$ & 3,30 & & \\
\hline & Total & 16,67 & 15,00 & $-1,67$ & 3,88 & & \\
\hline
\end{tabular}

$* \mathrm{p}<0,05$. 


\section{INTERVENCIÓN PSICOLÓGICA FAMILIAR EN LA ENFERMEDAD DE ALZHEIMER}

Como se puede observar en la tabla 3, se han encontrado diferencias estadísticamente significativas con las variables "cuidador principal" y "Apoyo psicológico" $(p<0,05)$. Por otro lado, no se han podido establecer diferencias en la mejora de las puntuaciones del MEC cuando el familiar acude al programa "hablemos de Alzheimer" de los que no acuden. A continuación, en la tabla 4, se realizan análisis estadísticos para analizar las diferencias entre las características de las variables.

Tabla 4: HSD de Tukey con la variable cuidador principal.

\begin{tabular}{|l|l|c|c|c|}
\hline $\begin{array}{c}\text { (I) Núcleo de } \\
\text { convivencia }\end{array}$ & (J) Núcleo de convivencia & Diferencia de medias (I-J) & $\begin{array}{c}\text { Error } \\
\text { típico }\end{array}$ & Sig \\
\hline \multirow{2}{*}{ Cónyuge } & Otro familiar & 0,66 & 1,64 & 0,914 \\
\cline { 2 - 5 } & Residencia & $5,77^{*}$ & 1,50 & 0,003 \\
\hline \multirow{2}{*}{ Otro familiar } & Cónyuge & $-0,66$ & 1,64 & 0,914 \\
\cline { 2 - 5 } & Residencia & $5,11^{*}$ & 1,50 & 0,009 \\
\hline \multirow{2}{*}{ Residencia } & Cónyuge & $-5,77^{*}$ & 1,50 & 0,003 \\
\cline { 2 - 6 } & Otro familiar & $-5,11^{*}$ & 1,50 & 0,009 \\
\hline$* 0,05$.
\end{tabular}

Tal y como se mostró en la tabla 3, se encontró relación estadísticamente significativa entre la puntuación total del MEC y el núcleo de convivencia ( $p<0,01)$. Además de esta prueba, se realizó la prueba "HSD de Tukey" para relacionar las características de la variable. Se ha encontrado relación estadísticamente significativa cuando se relaciona "el cuidador principal es el cónyuge" y el centro residencia $(p<0,05)$; hay relación estadísticamente significativa cuando "el cuidador principal es otro familiar" y "el centro residencia" $(p<0,01)$ y, no se ha encontrado relación estadísticamente significativa entre el cuidador principal es el cónyuge y otro familiar. En la siguiente gráfica se muestra de manera más visual.

Gráfica 1: relación entre MEC y núcleo de convivencia.

Relación entre la evolución del MEC y el núcleo de convivencia

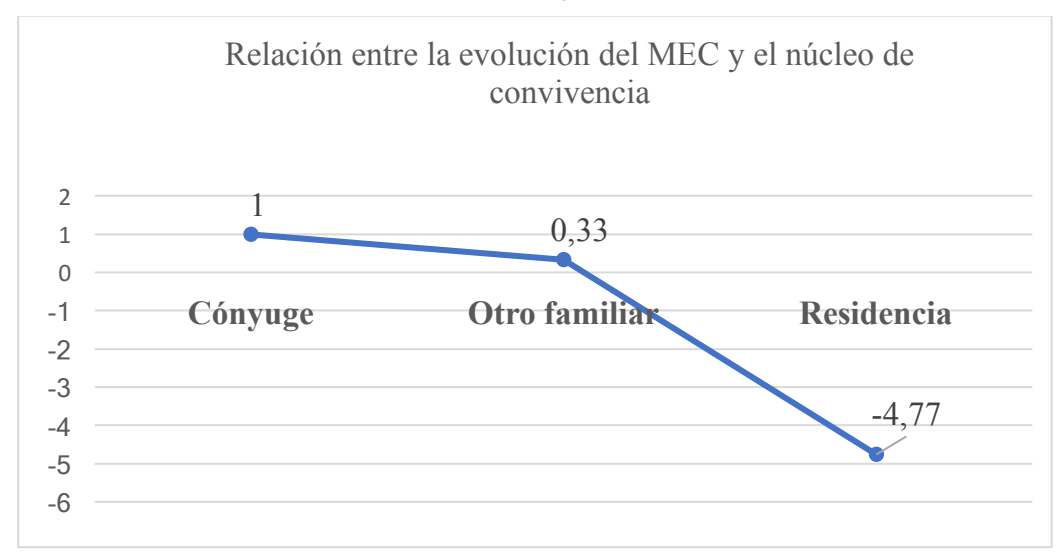

En la siguiente gráfica, se muestran las diferencias encontradas en la puntuación MEC antes y después de la aplicación del programa de estimulación relacionadas con la variable "apoyo psicológico", que se han encontrado diferencias estadísticamente significativas. 
Gráfica 2: relación entre MEC y apoyo psicológico a familiares.

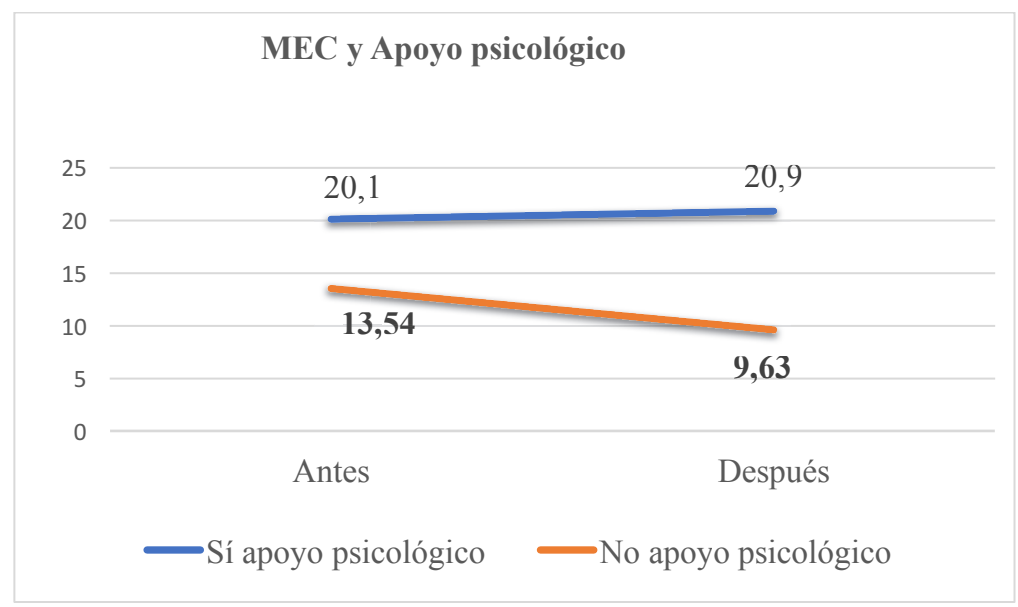

\section{DISCUSIÓN}

El objetivo principal de este estudio es comparar la eficacia de la aplicación de un programa de estimulación de seis meses de duración en pacientes con DTA sobre el rendimiento cognitivo. Los datos obtenidos indican que, la puntuación antes y después de la aplicación del programa de estimulación, en líneas generales ha mejorado. Esta mejoría obtenida en el MEC es similar a la encontrada en la literatura sobre la aplicación de un programa de estimulación en pacientes con DTA (López et al., 2020; Tárrega, 1998). Por otro lado, Ios sujetos con DTA sin la aplicación de un programa de estimulación, la media del rendimiento cognitivo general por año, fue de 4,2 puntos, puntuación que está muy por debajo de la encontrada en los estudios sobre la aplicación de los programas de estimulación (Yesavage y Sheikh, 1988). Por lo que, la aplicación de un programa de estimulación en DTA es efectiva si se compara con el grado de deterioro propio de la enfermedad. Sobre este punto, es conveniente tener en cuenta que la DTA es una enfermedad degenerativa primaria, por lo que, el objetivo en el que se basan los programas de estimulación es en el mantenimiento de la capacidad cognitiva y funcional del paciente. Teniendo en cuenta este objetivo, si el paciente manifiesta un mantenimiento (o mínima disminución) de la capacidad cognitiva y funcional general, es un éxito terapéutico tal y como se demuestra en este estudio.

La capacidad cognitiva general en la mayor parte de los estudios sobre la DTA, está valorada con el MMSE (Folstein et al., 1975). En este estudio la capacidad cognitiva general está valorada con el MEC (Lobo y Ezquerra, 1979). Salvando esta característica y teniendo en cuenta las limitaciones en cuanto al número total de sujetos estudiados, los resultados indican líneas interesantes de análisis.

En cuanto a las variables familiares, la tendencia observada en los datos de este estudio, indica que cuando Ios familiares de los pacientes con DTA acuden al programa "Hablemos de Alzheimer", la evolución de las capacidades cognitivas generales, se mantienen, mientras que los familiares que no acuden al programa, en los pacientes se observa una disminución de la capacidad cognitiva general, aunque no se han encontrado diferencias estadísticamente significativas. Cuando el cuidador principal del paciente con DTA solicita "apoyo psicológico", el paciente mantiene el rendimiento cognitivo general, mientras que los familiares que no lo solicitan, el rendimiento cognitivo general de los pacientes disminuye. Estos datos, relacionados con la modalidad de estancia, indican que los familiares de los pacientes en residencia solicitan mucha menor atención que los familiares en estancia diurna, por lo que los datos en esta línea están sesgados en cuanto a la modalidad de estancia (que se ha expuesto la diferencia significativa con relación a la evolución de la capacidad cognitiva general). Sobre 


\section{INTERVENCIÓN PSICOLÓGICA FAMILIAR EN LA ENFERMEDAD DE ALZHEIMER}

este dato, Tárraga (1998), manifiesta una central importancia al binomio paciente-cuidador. El tratamiento del entorno en igual medida que el paciente (se insiste en que el estudio de este autor se realizó en estancia diurna), por lo que es una variable esencial para tener en cuenta.

Con relación a la evolución de la capacidad cognitiva general y la relación con el cuidador principal, ha sido un dato para resaltar en este estudio. Este dato indica que cuando el cuidado se realiza en el centro residencial, se produce mayor deterioro que cuando el cuidador principal es un familiar o el cónyuge (en ambos casos se produce un mantenimiento). La relación encontrada en los dos últimos casos no es relevante, por lo que la diferencia en cuanto al cuidador va en la misma línea que la modalidad de estancia.

\section{CONCLUSIONES}

La aplicación del programa de estimulación cognitiva mantiene y mejora el rendimiento cognitivo general de los pacientes con DTA después de seis meses.

Cuando el trabajo se extiende al binomio familia-enfermo, mediante la aplicación de un tratamiento paralelo no farmacológico a los familiares, los resultados son diferentes en relación a la utilización del servicio de "apoyo psicológico" donde produce una mejoría significativa en el rendimiento cognitivo general y específicamente en el área de lenguaje y construcción; por otro lado, cuando el cuidador principal acude al programa "hablemos de Alzheimer", no se han encontrado diferencias estadísticamente significativas en relación al rendimiento cognitivo general.

El núcleo de convivencia del sujeto tiene un grado de significación, si el cuidador principal es el cónyuge u otro familiar, la evolución del rendimiento cognitivo general es mayor con relación a aquellos casos en los que los cuidados recaen en el centro residencial.

Una vez finalizado el estudio y los análisis necesarios, la premisa sobre la diferencia entre la curación y el cuidado se hace explícita. La DTA es una enfermedad neurodegenerativa sin tratamiento curativo en la actualidad. Con estudios como el presente, el objetivo no es la curación de la enfermedad, sino el cuidado del enfermo y del entorno familiar y social. Se ha pretendido demostrar con las limitaciones existentes en esta investigación, que el entorno tiene una influencia en la evolución del enfermo, el aspecto fundamental es la lucha contra la enfermedad y el cuidado de la persona, hasta que aparezca el tratamiento curativo.

\section{REFERENCIAS BIBLIOGRÁFICAS}

Agüera-Ortiz, L. F. (1998). Demencia: una aproximación práctica. Masson.

Agüera, L. F., Martín, M. M., y Durante, P. (2000). ¿Alzheimer? 100 preguntas más frecuentes. Edimsa.

Álvarez, C. (2002). El Alzheimer: La enfermedad y su entorno. Eneida.

Boada, M., Tárraga, L., y (Ed.). (1999). Volver a empezar: ejercicios prácticos de estimulación cognitiva para enfermos de alzheimer. Glosa.

Carrasco, M. M., Agüera Ortíz, L., Sánchez Ayala, M. I., Mateos Álvarez, R., Franco Martín, M., Castellano Arroyo, M., Villanueva Cañadas, E., Sánchez Pérez, M., y Cervilla Ballesteros, J. (2019). Consenso Español sobre Demencias (S. Sociedad Española de Psicogeriatría (ed.); $2^{\mathrm{a}}$ edición).

Cubero, P. (2002). Diagnóstico y tratamiento de las demencias en atención primaria. Masson.

De Shazer, S. (1989). Pautas de terapia familiar breve: un enfoque ecosistémico. Paidós.

European Observatory on Health Systems and Policies. (2019). State of Health in the EU España. Perfil sanitario nacional 2019 (pp. 1-24).

Folstein, M. F., Folstein, S. E., y McHugh, P. R. (1975). Mini-mental state": a practical method for grading the cognitive state of patients for the clinician. Journal of Psychiatric Research, 12(3), 189-198.

González-Mas, R. (2001). La enfermedad de alzheimer: clínica tratamiento y rehabilitación. Masson.

González-Rodríguez, V., y Martín-Martín, C. (2002). Demencias en atención primaria. Fomeco, 10(3), 128-150.

INE. (2018). España en cifras 2017. In Instituto Nacional de Estadística (p. 55). 
Lobo, A., y Ezquerra, J. (1979). El "mini-examen cognoscitivo": un test sencillo, práctico, para detectar alteraciones intelectivas en pacientes médicos. Actas Luso- Españolas de Neur y Psiquiatría, 3, 189-202.

López, C., Sánchez, J. L., y Martín, J. (2020). The effect of cognitive stimulation on the progression of cognitive impairment in subjects with Alzheimer's disease. Applied Neuropsychology: Adult, 1-10.

Martínez-Lage, P., Martín-Carrasco, M., Arrieta, E., Rodrigo, J., y Formiga, F. (2018). Mapa de la enfermedad de Alzheimer y otras demencias en España. Proyecto MapEA. Revista Espanola de Geriatria y Gerontologia, 53(1), 26-37. https://doi.org/10.1016/j.regg.2017.07.006

Orduna, G. ., y Naval, C. (2001). Gerontología educativa. Ariel educación.

Peña-Casanova, J. (1999). Colección "activemos la mente": libros 1,2,3,4. Fundación la Caixa.

Rodríguez-Sánchez, E., Mora-Simón, S., Patino-Alonso, M. C., García-García, R., Escribano-Hernández, A., García-Ortiz, L., Perea- Bartolomé, M. V., y Gómez-Marcos, M. A. (2011). Prevalence of cognitive impairment in individuals aged over 65 in an urban area: DERIVA study. BMC Neurology, 11. https://doi.org/10.1186/1471-2377-11-147

Tárrega, L. (1998). Terapias blandas: programa de psicoestimulación integral. Alternativa terapéutica para personas con enfermedad de Alzheimer. Revista de Neurología, 27(1), 51-62.

Thornton, H. A. (1989). A medical handbook for señor citizens and their families. Auburn House publishing Company.

Tola, M. (2018). El 55\% de los mayores de 65 años que tiene demencia no está diagnosticado. Https://Www.Ceafa.Es/Es/Que-Comunicamos/Noticias/El-55-de-Los-Mayores-de-65-Anos-Que-TieneDemencia-No-Esta-Diagnosticado. https://www.ceafa.es/es/que-comunicamos/noticias/el-55-de-los-mayores-de-65-anos-que-tiene-demencia-no-esta-diagnosticado

Vega Alonso, T., Miralles Espí, M., Mangas Reina, J. M., Castrillejo Pérez, D., Rivas Pérez, A. I., Gil Costa, M., López Maside, A., Arrieta Antón, E., Lozano Alonso, J. E., y Fragua Gil, M. (2016). Prevalence of cognitive impairment in Spain: The Gómez de Caso study in health sentinel networks. Neurologia. https://doi.org/10.1016/j.nrl.2016.10.002

Yesavage, J. A., y Sheikh, J. I. (1988). Nonpharmacologic treatment of age-associated memory impairment. Comprehensive Therapy, 14(6), 44-46. 
